\title{
SOME NEW PROPERTIES FOR THE RESOLVENT OPERATOR
}

\author{
ABDELLAH BNOUHACHEM
}

Abstract. The general mixed quasi variational inequalities (GMVI) are considerably difficult to solve directly, and hence, we often try to resolve this problem via solving some equivalent forms of GMVI. It is well known that GMVI are equivalent to the fixed point problems and resolvent equations. In this paper, we use these alternative equivalent formulations to suggest some important properties for the resolvent operator.

Mathematics subject classification (2000): 49J40, 65N30.

Key words and phrases: general mixed quasi variational inequalities, resolvent operator, fixed point problems, skew-symmetry.

\section{REFERENCES}

[1] H. BREZIS, Operateurs maximaux monotone et semigroupes de contractions dans les espace d'Hilbert, North-Holland, Amsterdam, Holland, 1973.

[2] R. Glowinski, J. L. LiOns AND R. Tremolieres, Numerical analysis of variational inequalities, North-Holland, Amsterdam, Holland, 1981.

[3] D. H. Hyers, G. IsaC And TH. M. Rassias, Topics in Nonlinear Analysis and Applications, World Scientific Publ. Co., Singapore, New Jersey, London, 1997.

[4] J. L. Lions, G. StAMPACCHIA, Variational inequalities, Comm. Pure Appl. Math., 20, (1967), $493-512$.

[5] M. A. Noor, General variational inequalities, Applied Math. Letters, 1, (1988), 119-121.

[6] M. A. NoOR, K. InAYAT NoOR AND TH. M. RASSIAS, SOME ASPECTS OF VARIATIONAL INEQUALITIES, Journal of Computational and Applied Mathematics, 47, (1993), 285-312.

[7] M. A. NOOR, K. INAYAT NOOR AND TH. M. RASSIAS, Set-valued resolvent equations and mixed variational inequalities, Journal of Mathematical Analysis and Applications, 220, (1998), 741-759.

[8] M. A. NoOR, TH. M. RASsias, Resolvent equations for set-valued mixed variational inequalities, Nonlinear Analysis - Theory, Methods and Applications, 42, (1) (2000), 71-83.

[9] M. A. Noor, Pseudomonotone general mixed variational inequalities, Appl. Math. Computation, 141, (2003), 529-540.

[10] M. A. Noor, Mixed quasi variational inequalities, Applied Mathematics and Computation, 146, (2003), 553-578.

[11] M. A. Noor, Some developments in general variational inequalities, Appl. Math. Computation, 152 , (2004), 199-277.

[12] M. A. Noor, K. I. Noor, On general mixed quasi variational inequalities, J. Optim. Theory Appl., 120, (3) (2004), 579-599.

[13] G. StAmPACCHia, Formes bilineaires coercitives sur les ensembles convexes, Comptes Rendues de 1' Academie des Sciences, Paris, 258, (1964), 4413-4416. 